\title{
SCANNING ELECTRON MICROSCOPIC EVALUATION OF THE SEALER/DENTINE INTERFACE OF TWO SEALERS USING TWO PROTOCOLS OF IRRIGATION
}

\author{
Elvira Gyulbenkiyan, Angela Gusiyska, Radosveta Vassileva, Elena Dyulgerova \\ Department of Conservative Dentistry, Faculty of Dental Medicine, Medical \\ University - Sofia, Bulgaria.
}

\section{ABSTRACT}

Purpose: To evaluate with scanning electron microscopy the sealing ability of the bioceramic sealer Total Fill BC and the resin-based sealer AH Plus using two protocols of irrigation.

Materials and Methods: All teeth $(n=40)$ were prepared using a crown-down technique with rotary nickel-titanium instruments. Two irrigation protocols are used for the purpose of this in vitro study: first (classical) protocol $10 \mathrm{ml} \mathrm{2.0 \%} \mathrm{NaOCl,} 10 \mathrm{ml} \mathrm{17 \%} \mathrm{EDTA} \mathrm{and} 10 \mathrm{ml}$ saline, and second (modified) protocol - $10 \mathrm{ml} 2.0 \% \mathrm{NaOCl}, 3 \mathrm{ml} 0.6 \%$ chitosan-citrate and $10 \mathrm{ml}$ saline. All teeth were allocated into four groups: group I $(n=10)$ - the first (classical) irrigation, obturation with AH Plus; group II $(n=10)$ - the second (modified) irrigation protocol, obturation with AH Plus; group III $(\mathrm{n}=10)$ - the first (classical) irrigation protocol, obturation with Total Fill and group IV $(n=10)$ - the second (modified) irrigation protocol, obturation with Total Fill BC. The adaptation of the sealer in the sealer/dentin interface (using SEM) was analyzed as the gap between the sealer and the dentine wall in $2 \mathrm{~mm}$ from the apex of the tooth was measured in microns.

Results: There was a statistically significant difference between groups with the different protocol of irrigation.

Conclusion: It was found that the application of chitosan-citrate $0.6 \%$ as final irrigating solution contributes high sealing ability both of the premixed bioceramic sealer Total Fill BC and the epoxy resin-based sealer $\mathrm{AH}$ Plus.

Keywords: bioceramic sealer, resin-based sealer, apical microleakage, chitosan-citrate

\section{INTRODUCTION}

The prevention of microbial contamination of the complex root canal system is essential for achieving a successful endodontic outcome - both clinical and radiographic. A wide variety of different endodontic filling materials have been developed for achieving a good sealing ability to the root canal dentin. In the scientific literature is reported that an ideal root canal sealer should adhere firmly to both dentin and core filling materials [1]. Sealing ability depends on the resistance of the material to the microleakage through its entire thickness. The good adaptation and strong bond of the endodontic sealer to the dentin are of great importance for preserving the integrity of the obturation material [2]. Various adhesive filling systems have been introduced in endodontics in an attempt to obtain a "monoblock" in which the core material, sealing agent and root canal dentin form a single cohesive unit within the root canal [3].

Chitosan is a natural linear polysaccharide obtained by the deacetylation of chitin, which is found in crab and shrimp shells. Biocompatibility, biodegradability, bioadhesion and lack of toxicity of chitosan are of great importance in dental medicine. Due to its high chelating ability for various metal ions in acidic conditions, it has been applied widely in the removal or recovery of metal ions in different industrial areas. The structure of chitosan is similar to that of extracellular matrix proteins, such as proteoglycans and glycosaminoglycan. Previous research demonstrated its ability to enhance the mechanical properties of dentin collagen and to reinforce collagen constructs [4]. Some studies showed that chitosan and their derivatives interacted with and neutralized matrix metalloproteinases (MMPs), which improved the resistance of dentin to degradation $[5,6]$. The chitosan-based irrigants showed a satisfactory ability to remove the smear layer and open the dentin tubules for subsequent sealing of the root canal system [4]. As a consequence of SEM investigation with different concentrations of chitosan-citrate (from $0.1-0.6 \%$ ), the chitosan-based irrigant solution (at a concentration of $0.6 \%$ ) showed a satisfactory ability to remove the smear layer and open the dentin tubules for subsequent sealing of the root canal system [4].

AH Plus (Dentsply Maillefer, Belgium, Switzerland) is an epoxy resin-based sealer, which used with gutta-percha is suitable for lateral and vertical condensation techniques. This sealer showed better long-term sealing ability compared to conventional sealers due to its reported expansion over time. The sealer has excellent biocompatibility as the risk of a postoperative inflammatory response is minimal [7].

Total Fill BC ( $F K G$, Germany) is a bioceramic sealer recognized for its biological properties such as biocom- 
patibility, bioactivity and antibacterial activity, as well as for its excellent physic-chemical properties $[8,9,10]$. Unlike conventional filling cements, the setting reaction of Total Fill BC ( $F K G$, Germany) is triggered by the moisture present in the dentinal tubules. Using this moisture, Total Fill BC sealer forms hydroxyapatite, to ensure optimum chemical adhesion between the dentin and the cement [11].

The study hypothesis was that the use of chitosancitrate solution $0.6 \%$ as a final irrigant could contribute to better adhesion of both bioceramic and epoxy resin-based sealer to the root canal dentine.

\section{Aim}

To compare and evaluate with scanning electron microscopy (SEM) the sealing ability of the bioceramic sealer Total Fill BC and the resin-based sealer AH Plus using two protocols of irrigation - classic and modified with chitosan-citrate $0.6 \%$ as a final irrigating solution.

\section{MATERIALS AND METHODS}

\section{Sample selection and preparation}

Forty one-rooted human teeth $(n=40)$ were used for the purpose of this in vitro study. All teeth were mechanically cleaned of any dental deposits and/or calculus by curettes and stored in saline at $4^{\circ} \mathrm{C}$ temperature. All teeth were selected with straight root canals - type I based on Vertucci's classification [12]. Before root canal preparation the crown was cut in the zone of the cement-enamel junction (CEJ), and the root length was standardized about $12 \mathrm{~mm}(+/-1$ $\mathrm{mm}$ ). The working length (WL) is standardized with K-file \#10 per $1 \mathrm{~mm}$ from the apex of the tooth as the instrument is inserted into the root canal until its tip is slightly protruded from the apex of the tooth and then was reduced with $1 \mathrm{~mm}$. The tips of the roots are sealed with sticky wax for maximum simulation of the clinical situation [13]. All teeth were prepared using a crown-down technique with hand steel instruments K-files \#10 and \#15 (Dentsply, Maillefer, Switzerland) and with rotary nickel-titanium instruments ProTaper Universal (Dentsply, Maillefer, Switzerland). The final preparation of the root canals is with file F4 (ProTaper Universal, Dentsply, Maillefer, Switzerland).

Two irrigation protocols are used for the purpose of this in vitro study.

- first (classical) protocol - $10 \mathrm{ml} 2.0 \% \mathrm{NaOCl}, 10$ $\mathrm{ml} 17 \%$ EDTA and $10 \mathrm{ml}$ saline

- second (modified) protocol - $10 \mathrm{ml} 2.0 \% \mathrm{NaOCl}, 3$ $\mathrm{ml} 0.6 \%$ chitosan-citrate and $10 \mathrm{ml}$ saline

Preparation of solution chitosan-citrate $0.6 \%$

A method for preparation of solution chitosan-citrate $0.6 \%$ has been developed. Low molecular weight chitosan (with high bioadhesive capacity) was used as $0.6 \mathrm{mg}$ of chitosan was dissolved in $100 \mathrm{~mL} 1 \%$ citric acid. The selected concentrations of both reagents fulfil the requirements for dissolving chitosan at $\mathrm{pH} 4.5$.

All teeth are randomly allocated into four groups:

Group I ( $\mathrm{n}=10)$ - the first (classical) irrigation protocol was applied and the root canals were obturated with AH Plus (Dentsply DeTray, Germany);

Group II $(\mathrm{n}=10)$ - the second (modified) irrigation protocol was applied, and the root canals were obturated with AH Plus (Dentsply DeTray, Germany);

Group III $(\mathrm{n}=10)$ - the first (classical) irrigation protocol was applied, and the root canals were obturated with Total Fill BC ( $F K G$, Germany);

Group IV ( $\mathrm{n}=10)$ - the second (modified) irrigation protocol was applied, and the root canals were obturated with Total Fill BC ( $F K G$, Germany);

All root canals were obturated with single cone technique using standardized gutta-percha cones and with the specified for the group sealer. After the root canal obturation, all teeth were coronary obturated with the composite material Filtek Z250 (3M ESPE, USA). Finally, all samples were imbedded in a wet sponge which simulated the periapex and forced the hardening process. (SEM)

Sample preparation for scanning electron microscopy

The teeth were split longitudinally using a diamond bur under water cooling. The specimens were then dehydrated by soaking in ethanol and were mounted on aluminum stubs, sputter coated with gold-argon (JEOL JFC-1200 Fine Coater, Tokyo, Japan), and observed under a field emissionscanning electron microscope (JEOL JSM-5510, Tokyo, Japan) at magnification x 250 and 1000. The adaptation a of the sealer in the sealer/dentin interface was analyzed as the gap between the sealer and the dentine wall in $2 \mathrm{~mm}$ from the apex of the tooth was measured in microns $(\mu \mathrm{m})$ using Image-Pro Plus 6.0 software (Media Cybernetics, USA) (figure 1). The comparative statistical analyzes were performed with Bootstrap for Independent Samples t-test, SPSS 19 (IBM, USA).

Fig. 1. Measuring the gap in the sealer/dentin interface using the software program Image-Pro Plus 6.0

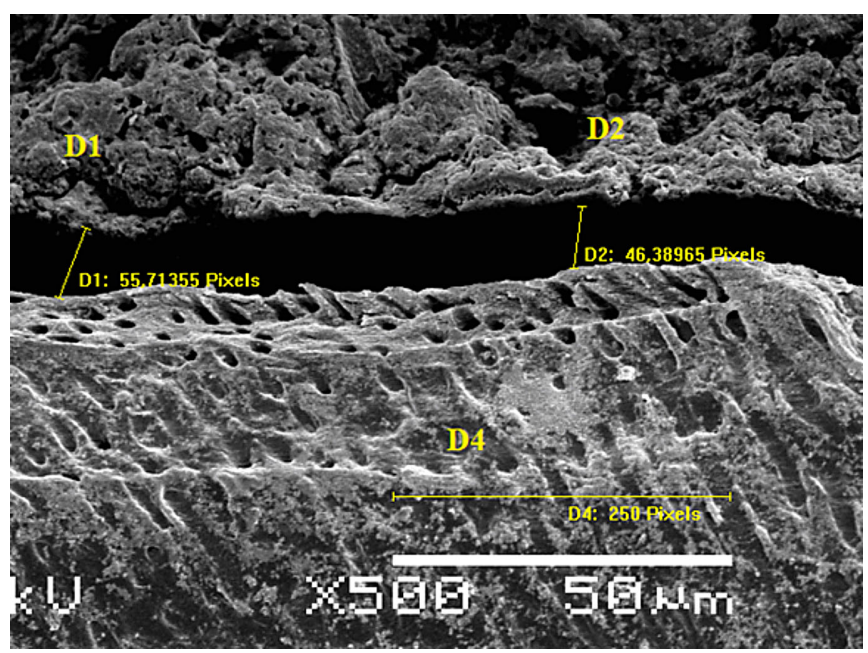

\section{RESULTS}

In group I the microleakage mean values of AH Plus and the classic protocol of irrigation were 12,51+/- 2,09. In group II (modified protocol of irrigation and obturation with AH Plus) mean values were 7,94 +/- 3,21. In group III (classic irrigation protocol and obturation with Total Fill BC) mean values were $11,96+/-4,29$ and in the last group IV (modified protocol of irrigation and obturation with Total Fill BC - 4,33+/- 1,56. (figure 2). 
Fig. 2. SEM analysis of apical microleakage in microns ( $\mu \mathrm{m})(\mathrm{SD}-\mathrm{Standard}$ Deviation)

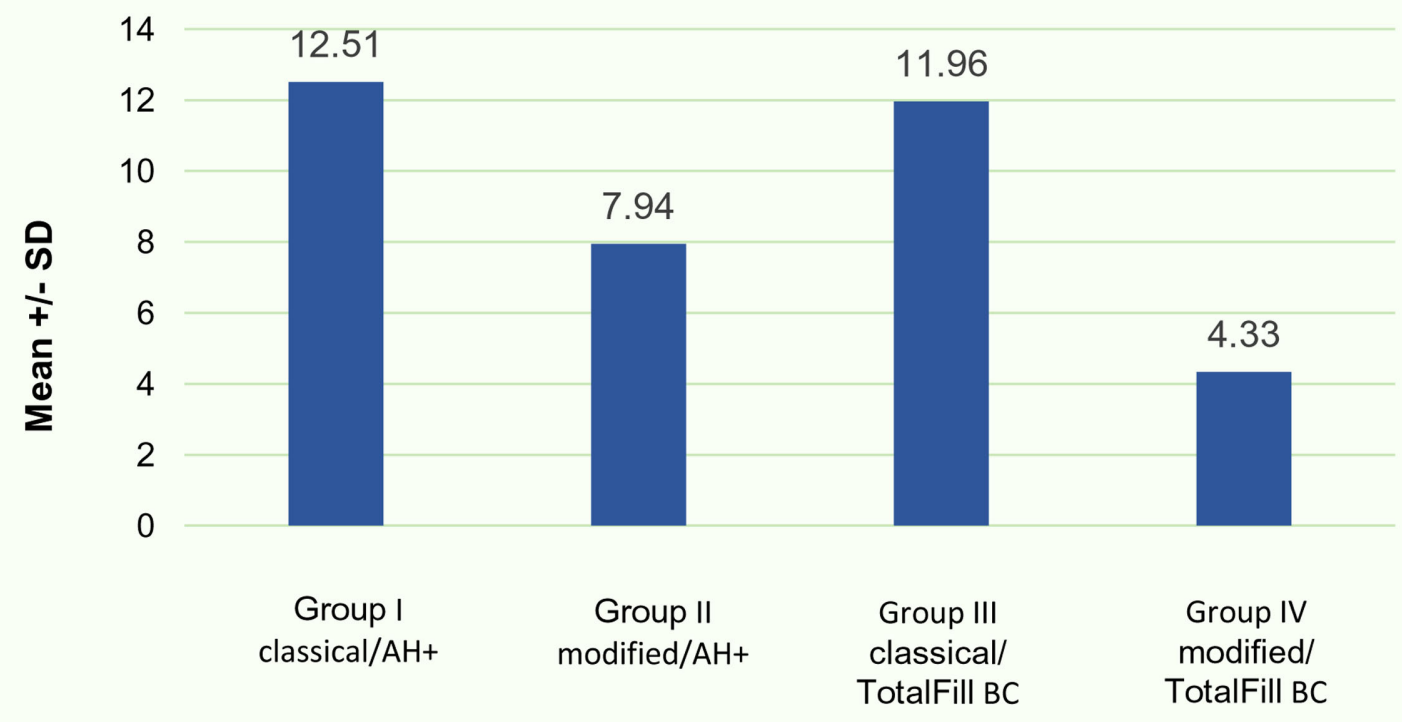

There was a statistically significant difference between the groups with the different protocol of irrigation (between group I and II $-p=0,033$, between group III and IV $-p=0,013)$. Between the groups with the different pro- tocol of obturation and the same protocol of irrigation it has been not determinate a significant change (between group I and III $-\mathrm{p}=0,806$ and between II and IV $-\mathrm{p}=0,054)$ (table $1)$.

Tab. 1. Comparison of the mean value of vertical apical microleakage between all groups

\begin{tabular}{|c|c|c|c|}
\hline I group & III group & I group & II group \\
\hline II group & IV group & III group & IV group \\
\hline $\mathrm{p}$ & $\mathrm{p}$ & $\mathrm{p}$ & $\mathrm{p}$ \\
\hline 0,033 & 0,013 & 0,806 & 0,054 \\
\hline
\end{tabular}

The following SEM images represent the adaptation of the sealer in the sealer/dentine interface in all four groups (figures $3-6$ ).

Fig. 3. SEM image of a longitudinal sectioned root canal, irrigated with the classic irrigation protocol, and obturated with AH Plus (magnification x 250)

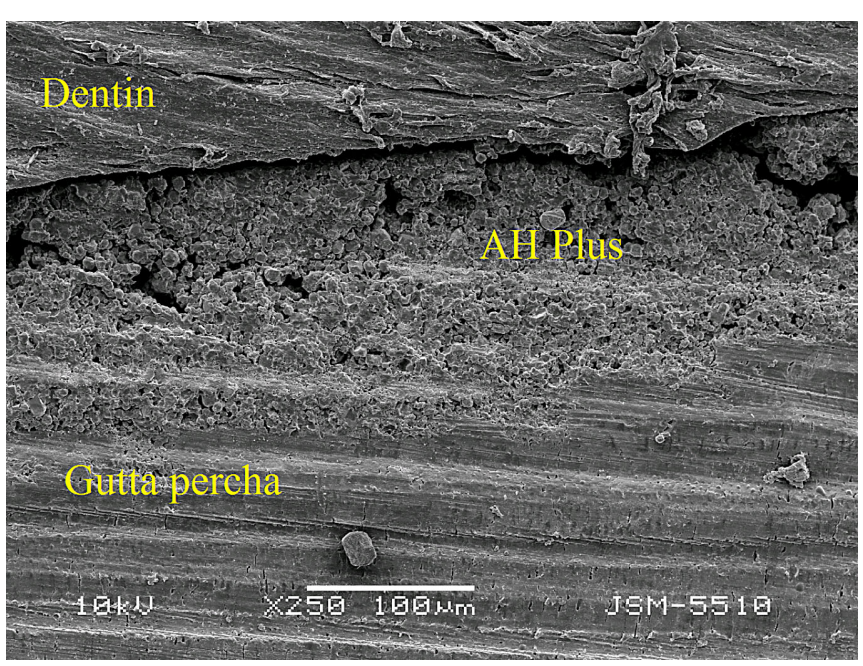

Fig. 4. SEM image of a longitudinal sectioned root canal, irrigated with the modified irrigation protocol, and obturated with AH Plus (magnification x 1000)

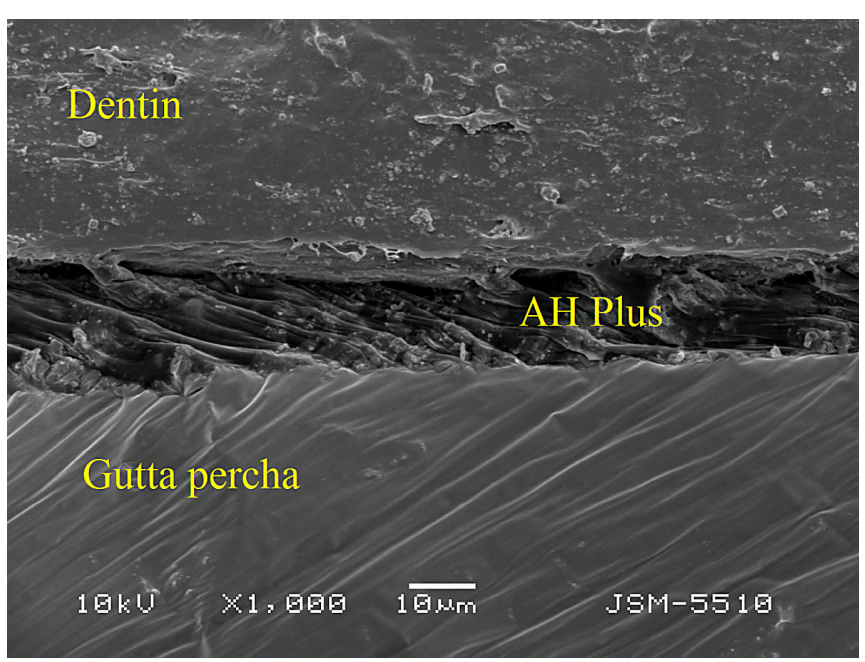


Fig. 5. SEM image of a longitudinal sectioned root canal, irrigated with the classic irrigation protocol, and obturated with Total Fill BC (magnification x 1000)

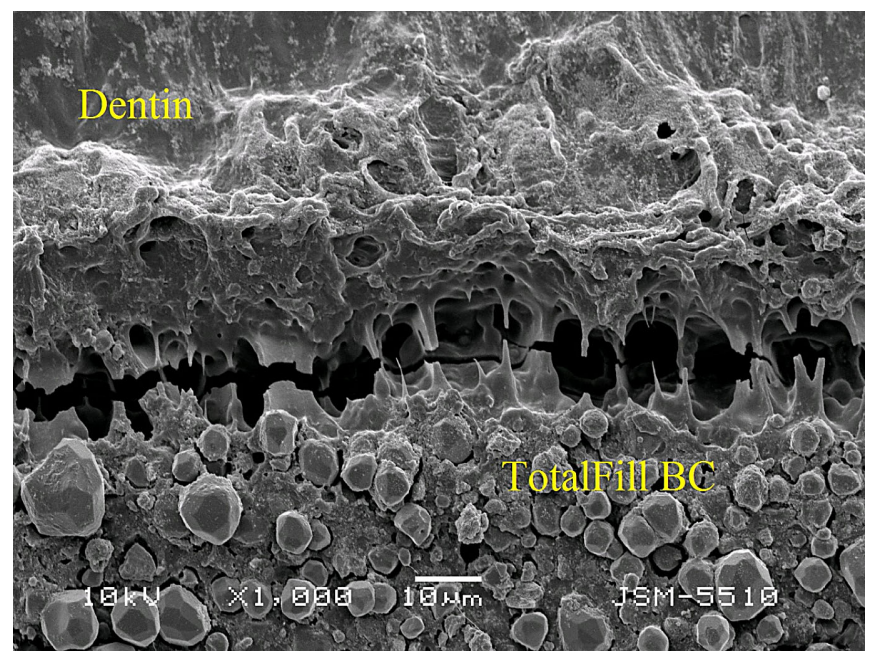

\section{DISCUSSION}

Eliminating the lack of possibility for microleakage of fluids, microorganisms and their by-products in the root canal system is a fundamental aim in endodontic treatment. After pulpectomy, root canal shaping and disinfection a high priority is the obturation material to achieve good adhesion to the dentine walls $[14,15]$. The microleakage - coronary, lateral and/or apical, is a major clinical problem that subsequently leads to failure of endodontic treatment. Endodontically treated teeth have significantly reduced mechanical strength due to creating endodontic access, the instrumental shaping of the root canals, as well as due to desiccation of dentine tissues [16, 17]. Various factors, such as root canal anatomy, the apical diameter, the chemical and mechanical preparation, the final irrigation and the degree of root canal contamination can be predispositions for microleakage into the root canals $[14,15]$.

In this in vitro study was analyzed the sealing possibility of two root canal sealers - an epoxy resin-based and a bioceramic sealer, using two irrigation protocols classic and modified (with chitosan- citrate solution $0,6 \%$ ), as all samples were observed with scanning electron microscopy. The bioceramic sealers are hydrophilic, they do not shrink and are insoluble in tissue fluids. Adhesion between bioceramic sealers and root canal dentine is influenced by the moisture present in the tooth. As the components of the bioceramic material enable the formation of calcium hydroxide and hydroxyapatite, they ensure an excellent bond to both the dentin and the filling material. Meanwhile they allow the practitioner to perform the microbial control without removing dentin unnecessarily and leaving a stronger root for restorative reconstruction [13]. A problem with hardening of premixed bioceramic sealers could be the lack of moisture in the root canal system, especially in already endodontically treated teeth [18].
Fig. 6. SEM image of a longitudinal sectioned root canal, irrigated with the modified irrigation protocol, and obturated with Total Fill BC (magnification x 250)

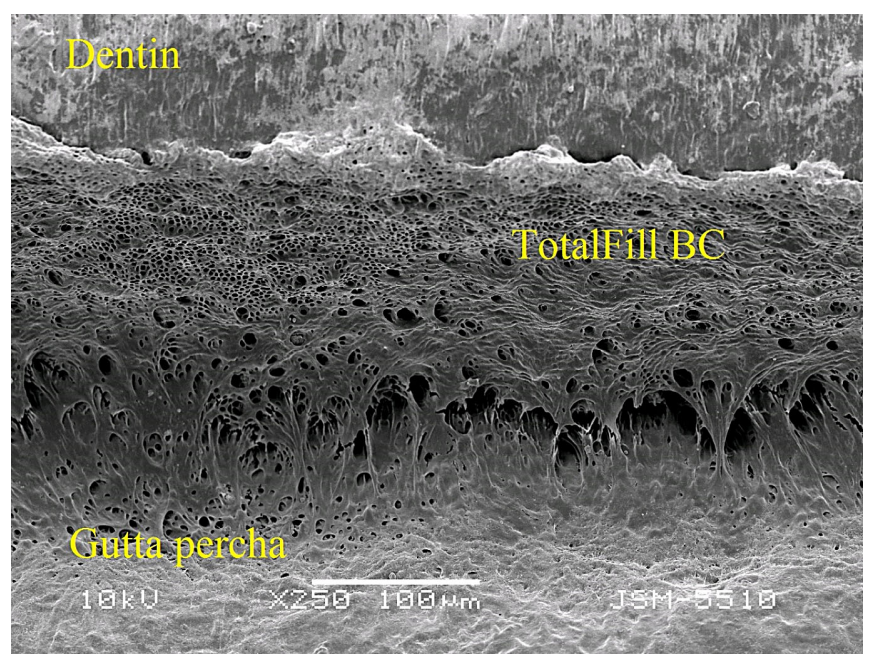

Epoxy resin-based sealer has good adhesive properties to the root canal dentin, have excellent biocompatibility and minimum risk of a postoperative inflammatory reaction. AH Plus has many advantages over other sealers, but the ability to ensure a hermetic root canal obturation is debatable in the literature $[7,19]$. The data from the SEM analysis distributed in the four groups in this in vitro study are as follows - in group I the microleakage mean values of $\mathrm{AH}$ Plus and the classic protocol of irrigation were 12,51 $+/-2,09$, in group II (modified protocol of irrigation and obturation with AH Plus) mean values were 7,94 +/- 3,21, in group III (classic irrigation protocol and obturation with Total Fill BC) mean values were 11,96+/- 4,29 and in the final group IV (modified protocol of irrigation and obturation with Total Fill BC - 4,33 +/- 1,56 (figure 2). Statistically, there was a significant change between the groups with the different protocol of irrigation (between group I and II $-p=0,033$, between group III and IV $-p=0,013$ ). Between the groups with the different protocol of obturation and same protocol of irrigation it has been not determinate a significant change (between group I and III $-\mathrm{p}=$ 0,806 and between II and IV $-\mathrm{p}=0,054$ ) (table 1).

In the study, we confirm the good sealing ability of both the bioceramic sealer Total Fill BC (mean 4,33 +/1,56) (figure 6) and the epoxy resin-based sealer AH Plus (mean 7,94+/- 3,21) (figure 4), using chitosan-citrate $0,6 \%$ as the final irrigating solution.

\section{CONCLUSION}

In the limitations of this in vitro study, it was found that the application of chitosan-citrate $0,6 \%$ as final irrigating solution contributes high sealing ability both of the premixed bioceramic sealer Total Fill BC and the epoxy resin-based sealer AH Plus. 


\section{REFERENCES:}

1. Abada HM, Farag AM, Alhadainy HA. Darrag AM. Push-out bond strength of different root canal obturation systems to root canal dentin. Tanta Dental Journal. 2015 Sep; 12(3):185-191. [Crossref]

2. Jafari F, Jafari S. Importance and methodologies of endodontic microleakage studies: A systematic review. J Clin Exp Dent. 2017 Jun 1;9(6): e812-e819. [PubMed] [Crossref]

3. Fisher MA, Berzins DW, Bahcall JK. An in vitro comparison of bond strength of various obturation materials to root canal dentin using a pushout test design. J Endod. 2007 Jul; 33(7): 856-8. [PubMed] [Crossref]

4. Gusiyska A, Vassileva R, Dyulgerova E, Ilieva R, Mironova J, Gyulbenkiyan E. Scanning electron microscopy studies of root canal dentin irrigated with a chitosan-citrate solution: a preliminary report. Int J Recent Sci Res. 2016 Jan;5(1):539542.

5. Kim MM, Kim SK. Chitooligosaccharides inhibit activation and expression of matrix metalloproteinase2 in human dermal fibroblasts. FEBS Lett. 2006 May 15;580(11):2661-6. [PubMed] [Crossref]

6. Pashley DH, Tay FR, Yiu C, Hashimoto M, Breschi L, Carvalho $\mathrm{RM}$, et al. Collagen degradation by host - derived enzymes during aging. J Dent Res. 2004 Mar;83(3):216-21.

\section{[PubMed] [Crossref]}

7. McMichen FR, Pearson G, Rahbaran S, Gulabivala K. A comparative study of selected physical properties of five root-canal sealers. Int Endod J. 2003 Sep;36(9):629-35. [PubMed] [Crossref]

8. Ma J, Shen Y, Stojicic S, Haapasalo M. Biocompatibility of two novel root repair materials. J Endod. 2011 Jun;37(6):793-8. [PubMed] [Crossref]

9. Mukhtar-Fayyad D. Cytocompatibility of new bioceramic-based materials on human fibroblast cells (MRC-5). Oral Surg Oral Med Oral Pathol Oral Radiol Endod. 2011 Dec; 112:e137-42. [PubMed] [Crossref]

10. Zhang W, Li Z, Peng B. Ex vivo cytotoxicity of a new calcium silicate-based canal filling material. Int Endod J. 2010 Sep;43(9):769-74. [PubMed] [Crossref]

11. Debelian G, Trope M. The use of premixed bioceramic materials in endodontics. G Ital Endod. 2016 Nov; 30(2):70-80. [Crossref]

12. Vertucci FJ. Root canal anatomy of the human permanent teeth. Oral Surg Oral Med Oral Pathol. 1984 Nov;58(5):589-99. [PubMed] [Crossref]

13. Darrag AM. Effectiveness of different final irrigation solutions on smear layer removal in intraradicular dentin. Tanta Dental Journal. 2014 Aug;11(2):93-99. [Crossref]
14. Goldberg M, Lacerda-Pinheiro S, Jegat N, Six N, Septier D, Priam F, et al. The impact of bioactive molecules to stimulate tooth repair and regeneration as part of restorative dentistry. Dent Clin North Am. 2006 Apr; 50(2):277-98. [PubMed] [Crossref]

15. Gusiyska A, Gyulbenkiyan E, Vassileva R, Dyulgerova E. Effective root canal irrigation - a key factor of endodontic treatment - review of the literature. Int J Recent Sci Res. 2016; 7(4):9962-9970.

16. Kishen A. Mechanics and risk factors for fracture predilection in endontically treated teeth. Endodontic Topics. 2006 Mar;13(1):57-83. [Crossref]

17. Lang H, Korkmaz Y, Schneider $\mathrm{K}$, Raab WH. Impact of endodontic treatments on the rigidity of the root. J Dent Res. 2006 Apr;85(4):364-8. [PubMed] [Crossref]

18. Candeiro GT, Correia FC, Duarte MA, Ribeiro-Siqueira DC, Gavini G. Evaluation of Radiopacity, $\mathrm{pH}$, Release of Calcium Ions, and Flow of a Bioceramic Root Canal Sealer. $J$ Endod. 2012 Jun;38(6):842-5. [PubMed] [Crossref]

19. Ørstavik D, Nordahl I, Tibballs JE. Dimensional change following setting of root canal sealer materials. Dental Materials. 2001 Nov;17(6): 512-9. [PubMed] [Crossref]

Please cite this article as: Gyulbenkiyan E, Gusiyska A, Vassileva R, Dyulgerova E. Scanning electron microscopic evaluation of the sealer/dentine interface of two sealers using two protocols of irrigation. J of IMAB. 2020 JanMar;26(1):2887-2891. DOI: https://doi.org/10.5272/jimab.2020261.2887

Received: 29/05/2019; Published online: 27/01/2020

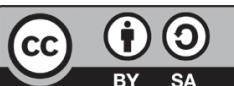

Address for correspondence:

Elvira Gyulbenkiyan

Department of Conservative Dentistry, Faculty of Dental Medicine, Medical Universitv - Snfia

1, Georgi Sofiyski Blvd., Sofia, Bulgaria.

E-mail: elvira.simon@gmail.com 